\title{
Thank You Peer Reviewers
}

Peer review is the cornerstone of the mission of ASHS journals - to advance the science of horticulture by publishing accurate, clear, reproducible research. Reviewers are the unsung heroes of that process.

To that end, we wish to express sincere appreciation to the scientists who have generously contributed their valuable time and expertise to assist both authors and editors in maintaining the high quality of HortTechnology.

The individuals listed below have provided reviews for one or more manuscripts during the past publication year. Their names are published here in recognition of their valuable contributions to ASHS and horticultural science. Every effort has been made to compile an accurate list from the records kept by the Society; any omissions are inadvertent.

Jeffrey Adelberg

Albert T. Adjesiwor

Shinsuke Agehara

Shayan Ali Akbar

Lisa Alexander

Hussein Ali Salim

Tom W. Allen

James E. Altland

Peter A.Y. Ampim

Sandra Anagnostakis

Neil O. Anderson

Jeanine Arana

Ramon A. Arancibia

Michael A. Arnold

Matt Arrington

Jatinder S. Aulakh

Gary R. Bachman

James H. Baird

Michael R. Barnes

Susan S. Barton

Richard Bastías

Pamela J. Bennett

Thierry E. Besancon

Harbans L. Bhardwaj

Guihong Bi

Cale Bigelow

Thomas Björkman

Brent Black

Eugene K. Blythe

Carlos E. Bogran

Arend-Jan Both

Cheryl R. Boyer

Jennifer Campbell Bradley

Terence L. Bradshaw

Rebecca N. Brown

Gerald Brust

David R. Bryla

Bruce Bugbee

Natalie R. Bumgarner

Stephanie Elaine Burnett

Rhoda L. Burrows

Tina M. (Waliczek) Cade
Michael D. Cahn

Benjamin Campbell

Julie H. Campbell

Matthew Chappell

Carlene A. Chase

Xuqi Chen

Yan Chen

Juang-Horng Chong

Nick Christians

Kelley Clark

Jon M. Clements

Joseph H. Connell

David S. Conner

Timothy Coolong

Warren E. Copes

Jeremy S. Cowan

David Coyle

Beatriz Cuenca

Christopher Jon Currey

Matthew A. Cutulle

Andre Luiz Biscaia Ribeiro

da Silva

Abdolmajid Mahdavi Damghani

Surendra K. Dara

Joan R. Davenport

Alejandro Del Pozo-Valdivia

Lisa Wasko DeVetter

Sadanand A. Dhekney

Juan Carlos Diaz Perez

Ryan W. Dickson

Maria Cecilia do Nascimento

Nunes

John M. Dole

Yuanhua Dong

Douglas . James Doohan

Sheri T. Dorn

João Paixão dos Santos Neto

Daniel Drost

J. Scott Ebdon

Mark K. Ehlenfeldt

Marianne Elliott

Coleman L. Etheredge
Esmaeil Fallahi

Jarrad Farris

James E. Faust

Louise Ferguson

Gina E. Fernandez

Rhuanito S. Ferrarezi

Jeb S. Fields

Charles Fontanier

Wheeler G. Foshee

Lorenzo Furlan

R. Karina Gallardo

Gary Yu Gao

Zhifeng Gao

Nicholas Genna

Lyn A. Gettys

Rahmatallah Gheshm

Stanton Gill

Leonard Githinji

Celina Gomez Vargas

Greta Gramig

Rebecca Grube Sideman

Mengmeng Gu

Sanjun Gu

Wenjing Guan

Daniel E. Guyer

Ivette Guzman

Howard Haldor

Frank A. Hale

Charles R. Hall

Xiaoyan Han

Camp Hand

Richard L. Harkess

Harlene M. Hatterman-Valenti

Cynthia L. Haynes

Joseph R. Heckman

Peter M. Hirst

William Hoch

Mark Hoffmann

Renee Holland

Emily E. Hoover

Tracy Hueppelsheuser

David R. Huff
Mark Hutchinson

Laura E. Irish

David Jespersen

A. Maxwell Jones

Shimat V. Joseph

Meriam G. Karlsson

Hayk Khachatryan

Mary Beth Kirkham

Roger Keith Kjelgren

Cynthia D. Klemmer

Wesley L. Kline

Melinda J. Knuth

Kent D. Kobayashi

Masakazu Komatsuzaki

Matthew H. Kramer

Heidi Kratsch

Helen Tyler Kraus

Chieri Kubota

Thomas P. Kuhar

Kristine M. Lang

Petrus Langenhoven

Anthony V. LeBude

Bernd Leinauer

Daniel Ivan Leskovar

Tongyin $\mathrm{Li}$

Robert Grant Linderman

Alexander G. Litvin

Guodong David Liu

Sally Logsdon

Natalie Lounsbury

Eliezer S. Louzada

Jessica Dawn Lubell-Brand

Robert E. Lyons

Shahla Mahdavi

Niels O. Maness

Ravishankar Manickam

S. Christopher Marble

Richard P. Marini

Jordan M. Marshall

Joseph G. Masabni

Felipe Barrios Masias

Elizabeth Maynard 
James (Jay) D. McCurdy

Garry Vernon McDonald

Ryan W. McEwan

Amy McFarland

Mica F. McMillan

Amanda L. McWhirt

Inga Meadows

Carmen Medina-Mora

Mary Hockenberry Meyer

Stephen L. Meyers

David Michener

Carol A. Miles

Amy C. Miller

Chad T. Miller

Gilbert Miller

Grady L. Miller

Gregory Miller

Elizabeth J. Mitcham

Susan C. Miyasaka

Odón Roberto Montes-

Colmenares

Kimberly A. Moore

J. Pablo Morales-Payan

Marcelo L. Moretti

Desmond G. Mortley

Yasser M.M. Moustafa

Mair Murray

James R. Myers

Lloyd L. Nackley

Kyle Nagy

Daniel Neuhoff

Mathieu Ngouajio

Genhua Niu

Marcin Nowicki

Angela Mary O'Callaghan

James W. Olmstead
Mercy A. Olmstead

Emmanuel C. Omondi

Robert Orpet

James S. Owen, Jr.

Cristi L. Palmer

Karen Panter

Babu Panthi

Robert E. Paull

Charles H. Peacock

Brian J. Pearson

Gregory M. Peck

H. Brent Pemberton

Svoboda Bodie Pennisi

Penelope M. Perkins-Veazie

Bryan J. Peterson

Benjamin Phillips

David M. Picha

Jeremy M. Pickens

Cristina Pisani

Bruno J.I. Pitton

Anne Plotto

Anissa Poleatewich

Robert Frank Polomski

Marvin P. Pritts

Michael Richardson

Chance W. Riggins

Alica Rihn

Sonia Rios

Mark A. Ritenour

Monique J. Rivera

Carolyn W. Robinson

James W. Rushing

Rossana Sallenave

Thomas J. Samples

Jayesh B. Samtani

German Sandoya
Umakanta Sarker

Joseph C. Scheerens

Marco Schiavon

Michael A. Schnelle

William J. Sciarappa

Chrissie Segars

Andrew F. Senesac

Matteo Serena

Kenneth A. Shackel

Travis W. Shaddox

Jacob Shreckhise

Alvin M. Simmons

Hardeep Singh

J. Robert Sirrine

Margaret Skinner

John Snyder

Douglas J. Soldat

Lynn M. Sosnoskie

Kayla A. Spawton

Eric Thomas Stafne

Leigh Anne Starling

Melanie N. Stock

Derrick Ryan Stowell

Bernadine Strik

Danuta Sugier

Youping Sun

Sven Svenson

Ashley Thompson

Grant L. Thompson

Peter R. Tozer

Danielle D. Treadwell

Daniel Tregeagle

Rebecca Turk

Mark E. Uchanski

Margarita Valendia

Arthur Villordon
Edgar L. Vinson

James T. Vogt

Kurt M. Vollmer

Russell W. Wallace

Matthew Wallhead

Kellie Jean Walters

Ajit Arun Waman

Yin-Tung Wang

Zinan Wang

Scott E. Warnke

Gary Watson

Sean Michael Westerveld

Sarah A. White

Cheryl A. Wilen

Kimberly Ann Williams

Sandra B. Wilson

Anthony L. Witcher

Jeffrey C. Wong

Samuel E. Wortman

Annette L. Wszelaki

Xuan (Jade) Wu

Mingying Xiang

Xi Xiong

Wei Qiang Yang

Joseph R. Young

Chengyan Yue

Bernard H. Zandstra

Qi Zhang

Xin Zhang

Xin Zhao

Jianfeng Zhou

Heping Zhu

Jared Zystro 